\title{
Hierarchical support vector data description for batch process monitoring
}

Zhaomin Lv, Xuefeng Yan ${ }^{*}$

Key Laboratory of Advanced Control and Optimization for Chemical Processes of Ministry of Education, East China University of Science and Technology, Shanghai 200237, P. R. China

*Corresponding Author: Xuefeng Yan

Email: xfyan@ecust.edu.cn

Address: P.O. BOX 293, MeiLong Road NO. 130, Shanghai 200237, P. R. China

Tell: 0086-21-64251036 


\section{Supporting Information}

The batch data $\boldsymbol{X}(I \times J \times K)$ is unfolded batch-wise, as illustrated in Figure 1. Each column in $\boldsymbol{X}_{B}(I \times J K)$ needs to be scaled to zero mean and unit variance matrix. $\overline{\boldsymbol{X}}_{B}(I \times J K)$ is then transformed into the form of a variable-wise matrix $\boldsymbol{X}_{V}(I K \times J)$. All data are illustrated in Figure 2 .

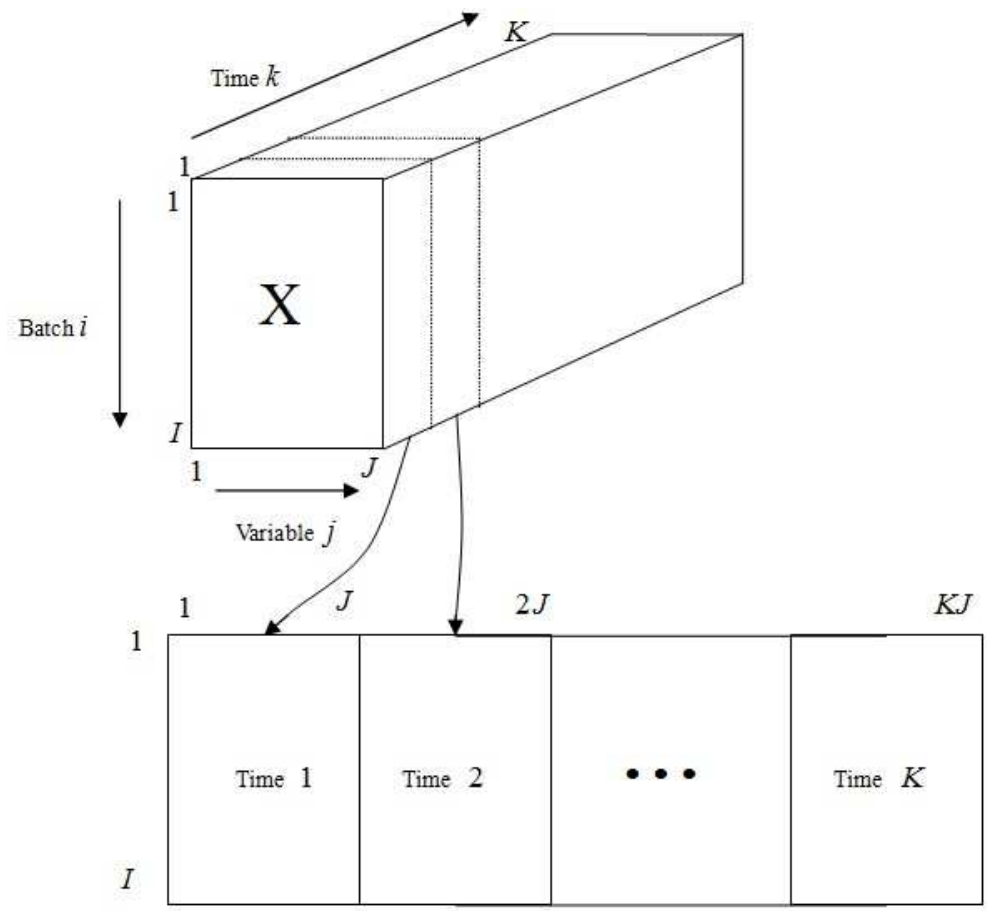

Figure 1. Batch-wise unfolding.

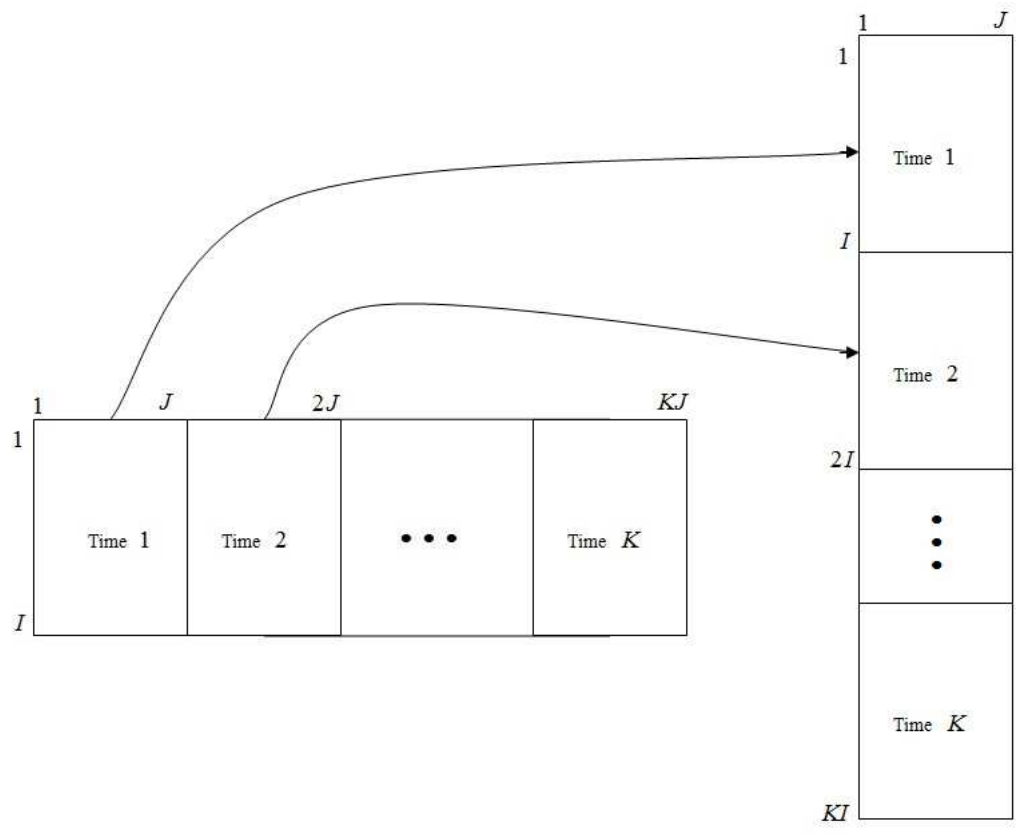


Figure 2. Batch-wise unfolding transformed to variable-wise unfolding 